\title{
Defining the Mechanism of Subarachnoid Hemorrhage-Induced Pyrexia
}

\author{
Ajith J. Thomas ${ }^{1} \cdot$ Luis Ascanio-Cortez $^{1} \cdot$ Santiago Gomez ${ }^{1} \cdot$ Mohamed Salem $^{1} \cdot$ George Maragkos $^{1} \cdot$ Khalid A. Hanafy $^{2}$ \\ Published online: 5 May 2020 \\ (C) The American Society for Experimental NeuroTherapeutics, Inc. 2020
}

\begin{abstract}
Fever can affect the majority of patients with subarachnoid hemorrhage (SAH) and many times no identifiable source is found for the fever whether infectious or sterile, like deep vein thrombosis. We hypothesized that fever in SAH is mediated by a NONcyclo-oxygenase-dependent mechanism, which we neologized as subarachnoid hemorrhage-induced pyrexia (SAHiP). This hypothesis was investigated using genetically modified mice, pharmacological manipulation, cerebrospinal fluid from SAH patients, and a large cohort of SAH patients. Mice with deletions of neuronal prostaglandin EP3 receptor, global toll-like receptor 4 (TLR4), myeloid TLR4, and microglial TLR4 were subjected to SAH after being implanted with thermometers. Pathways necessary for SAHiP were identified. In SAH patients, cerebrospinal fluid was examined by flow cytometry and correlated with SAHiP. From a large cohort of SAH patients, independent associations with SAHiP were determined using logistic regression analysis. In our mouse model of SAH, microglial TLR4 is necessary for SAHiP, but independent of the neuronal prostaglandin EP3 receptor, cyclo-oxygenase, and prostaglandins. Macrophages from the cerebrospinal fluid of SAH patients with SAHiP expressed more TLR4-co-receptor than SAH patients without SAHiP. In a large cohort of SAH patients, SAHiP was found to be independently, yet inversely, associated with acetaminophen administration. SAHiP is independent of the neuronal prostaglandin EP3 receptor, cyclo-oxygenase, and prostaglandins, but dependent on microglial/macrophage TLR4 with evidence from both SAH mouse models and SAH patients.
\end{abstract}

Keywords Microglia $\cdot$ Myeloid cells $\cdot$ Pyrexia $\cdot$ CSF macrophages $\cdot$ SAH $\cdot$ SAHiP $\cdot$ Prostaglandin-Independent

\section{Introduction}

Fever occurs in the majority of patients in a neurological intensive care unit (Neuro-ICU), and can affect up to $70 \%$ of patients [1-5]. Interestingly, many of the Neuro-ICU patients that present with fever do not have an identifiable source for the fever [6].

Fevers without an identifiable source have been clinically investigated mostly in relation to aneurysmal subarachnoid hemorrhage (SAH), where it is commonly seen, with varying

Electronic supplementary material The online version of this article (https://doi.org/10.1007/s13311-020-00866-x) contains supplementary material, which is available to authorized users.

Khalid A. Hanafy

khanafy@bidmc.harvard.edu

1 Department of Surgery, Beth Israel Deaconess Medical Center, Harvard Medical School, Boston, MA, USA

2 Department of Neurology, Division of Neurointensive Care, Beth Israel Deaconess Medical Center, Boston, MA, USA definitions and monikers for this type of fever. That being said, all fever comes from cerebral output that involves hypothalamic relays, most specifically located in the median preoptic nucleus (MnPO) utilizing the prostaglandin EP3 receptor; making terminology like neurogenic fever or central fever redundant [7-10]. The generation of prostaglandins requires the enzyme, cyclo-oxygenase (COX), to catalyze the conversion of arachidonic acid to prostaglandins which initiate the febrile response by activating the EP3 receptor on the $\mathrm{MnPO}$. The use of COX inhibitors, such as acetaminophen or meloxicam, is based on the premise that prostaglandins are required for a febrile response. In fact, the use of COXinhibitors to control the fever in SAH has failed many times in large clinical trials, suggesting that the mechanism behind these fevers, which we call subarachnoid hemorrhageinduced pyrexia (SAHiP), may be different [11-13].

In this study, we hypothesized that SAHiP occurs independently of the prostaglandin receptor pathway, and we tested this hypothesis using both genetic and pharmacological approaches. Genetically, mice with a conditional deletion of the neuronal 
EP3 receptor were subjected to SAH, and SAHiP was measured. Pharmacologically, wild-type mice subjected to SAH were treated with a COX inhibitor after SAH, and SAHiP was measured.

We also hypothesized that microglial TLR4 is necessary for SAHiP. We explored this hypothesis using mice with a global deletion of toll-like receptor 4 (TLR4), mice with a conditional deletion of all myeloid TLR4, and mice with a conditional deletion of microglial TLR4; all these mice were subjected to SAH and SAHiP was measured.

We also hypothesized that the expression of TLR4 moieties on macrophages from the cerebrospinal fluid of SAH patients would be associated with SAHiP and that in a large cohort of SAH patients we could determine independent associations with SAHiP.

\section{Materials and Methods}

Animal Information and Anesthesia All experimental procedures were approved by the Institutional Animal Care and Use Committee (IACUC) at Beth Israel Deaconess Medical Center (BIDMC). The facility is accredited by the Association for Assessment and Accreditation of Lab Animal Care and fully complied with all federal, state, and local laws. Animals were housed at BIDMC and fed a standard rodent diet ad libitum with 24-h access to either water and/or hydrogel while kept on a 12-h light/12-h dark cycle at $24{ }^{\circ} \mathrm{C} \pm 1.5^{\circ} \mathrm{C}$. All surgical manipulations were performed under general anesthesia with a $10 / 4 \mathrm{mg} / \mathrm{kg}$ mixture of ketamine (VetOne) and xylazine (Akorn Animal Health), respectively. For procedures, 8-12week-old male and female mice, all on a C57BL/6 CD 45.2 $2^{+}$ background, were obtained from the Jackson Laboratory unless otherwise indicated: B6.129P2 $(\mathrm{Cg})-\mathrm{Cx} 3 \mathrm{cr} 1^{\text {tm2.1(cre/ }}$ ERT2)Litt $\left(\mathrm{CX}_{3} \mathrm{Cr} 1^{\mathrm{CreER}}\right.$, stock no. 021160-a tamoxifeninducible myeloid-driven Cre promoter), B6.129P2Lyz $2^{\text {tml(cre)Ifo }}\left(\mathrm{LyzM}^{\mathrm{Cre}}\right.$, stock no. 004781 - a myeloid driven Cre promoter), B6.Cg-Tg(Nes-cre) $1 \mathrm{~K} l n$ (Nestin $^{\text {Cre }}$, stock no. 003771 - an early astrocyte and neuron-driven Cre promoter), B6(Cg)-Tlr $4^{\text {tm 1.1 Karp }}$ (TLR4 $4^{\mathrm{fl} / \mathrm{fl}}$, courtesy of Dr. Tim Billiar), B6;129-Ptger $3^{\text {tm } 1 C s m l}$ (ptger $3^{\mathrm{fl} / \mathrm{fl}}$, courtesy of Dr. Michelle Kimple, University of Wisconsin Madison), B6(Cg)Tlr4 ${ }^{\text {tm 1.2Karp }}$ (TLR4 ${ }^{-/-}$, stock no. 029015), and B6.SJLPtprc $^{a}$ Pepc $^{b}$ (C57BL/6 CD45.1 ${ }^{+}$mice, stock no. 002014). The following murine crosses were generated: $\mathrm{CX}_{3} \mathrm{Cr} 1^{\mathrm{CreER}}: \mathrm{TLR}^{\mathrm{fl} / \mathrm{fl}}$ (tamoxifen-inducible to create a microglial specific knockout of TLR4), LyzM ${ }^{\mathrm{Cre}}: \mathrm{TLR}^{\mathrm{fl} / \mathrm{fl}}$ (TLR4 knockout on all myeloid cells), and Nes ${ }^{\mathrm{Cre}}$ :ptger $3^{\mathrm{fl} / \mathrm{fl}}$ (a neuron and astrocyte-specific knockout of the prostaglandin EP3 receptor). All mice received $4 \mathrm{mg}$ of a $0.02 \mathrm{mg} / \mathrm{uL}$ subcutaneous injection of tamoxifen (Sigma-Aldrich) prepared in corn oil 1 week prior to placement of intraperitoneal thermometers (datalogger), so as to control for potential effects of tamoxifen upon body temperature. The research personnel that performed the surgical procedures were different from the personnel that conducted the temperature analysis or flow cytometry, thereby ensuring appropriate blinding.

\section{Surgical Procedures}

Laparotomy Procedure was performed for the placement of dataloggers (Subcue Minitemperature DataloggersTM, Canadian Analytical Technologies) that recorded the core temperature at 15 -min intervals. The mice were anesthetized as previously described, followed by a midline abdominal incision of approximately $2.5 \mathrm{~cm}$ in length, for placement of the datalogger. Primary closure of the abdominal wall, along with secondary skin closure, was performed using 5.0 silk suture.

SAH One week following datalogger implantation, mice were anesthetized and SAHs were induced as previously described [14]. Briefly, $60 \mu \mathrm{l}$ of arterial blood from donor CD $45.1^{+}$ mice, or $60 \mu \mathrm{l}$ of normal saline for controls, was injected at a $40^{\circ}$ ventral angle through a burr hole $4.5 \mathrm{~mm}$ anterior to the bregma to generate an SAH.

Intracerebroventricular Injection Intracerebroventricular injection was performed on anesthetized mice using a standard stereotaxic instrument setup (KOPF Instruments, Tujunga, CA, USA). One 2.5-mm-deep burr hole was drilled $0.22 \mathrm{~mm}$ posterior and $1 \mathrm{~mm}$ lateral to the bregma to enter the ventricle. One 5- $\mu \mathrm{g}$ injection of lipopolysaccharide (LPS) at a concentration of $1 \mu \mathrm{g} / \mu \mathrm{l}$ was administered using premeasured capillaries, as previously described $[14,15]$.

Fever Burden Calculation Dataloggers were removed following euthanasia and temperature recordings were recovered. Fever burden was calculated by plotting the difference in core temperature, per reading, relative to the temperature prior to $\mathrm{SAH}$, in a time-locked fashion. A total of 300 measurement differentials (15-min intervals), or 3.125 days, were summed to yield the fever burden.

Flow Cytometry and Antibodies Murine cells isolated from whole brain or blood were re-suspended in FACS buffer (1\% bovine albumin, $2 \mathrm{mM}$ EDTA), and $0.05 \% \mathrm{NaN} 3$ in PBS. Non-specific binding was blocked by treatment with anti-CD16/32 Trustain FcX (1:100; Biolegend) prior to incubation with antibodies directed against the following antigens: CD45.1-BV510, CD45.2-BV785, CD11b-PE/Dazzle, CD11c-APC, $\mathrm{CX}_{3}$ Cr1-FITC, CD68-AF700, GR-1-PE/Cy7, TLR4-PE, and Tmem119-AF790 (1:100; BioLegend). Endogenous cells were identified by exclusion of donor CD $45.1^{+}$cells. Blood from CD $45.1^{+}$mice was used to differentiate the injected blood from among the endogenous leukocytes of CD $45.2^{+}$experimental SAH mice. The highly 
specific microglial marker, Tmem119 [16], was used to identify CD $45.2^{+} \mathrm{CD} 11 \mathrm{~b}{ }^{\text {hi }} \mathrm{CX} 3 \mathrm{CR} 1{ }^{\text {hi }} \mathrm{CD} 68^{\text {int }} \mathrm{Tmem} 119^{\text {hi }}$ endogenous microglia. Blood-circulating monocytes and neutrophils were identified by size and granularity (forward- and sidescatter), and validated by $\mathrm{CD} 11 \mathrm{~b}^{\mathrm{hi}} \mathrm{CD} 68^{\mathrm{hi}}$ and $\mathrm{Gr}-\mathrm{1}^{\mathrm{hi}}$ signatures, respectively. Appropriate unstained controls were used to determine positive populations. For analysis of human CSF, non-specific block was followed by incubation with antibodies directed against CD15-PeCy7, and CD14-APC (5:100, Biolegend). CSF macrophages were classified as $\mathrm{CD} 14^{+} \mathrm{CD} 15^{-}$. The murine flow cytometry acquisition was performed on a CytoFLEX LX (BD Biosciences) and human CSF data was acquired on a FACSAria II (BD Biosciences). Analysis of all flow data was completed using FlowJo software (FlowJo, LLC.).

CSF Collection and Cell Pellet Harvest CSF (20 cc) from SAH patients was collected sterilely via the most distal external ventriculostomy drain (EVD) port within $48 \mathrm{~h}$ of ictus. The collected CSF was transported on ice and immediately processed. The CSF was centrifuged at $500 \times g$ for $5 \mathrm{~min}$ and the cell pellet was immediately processed for flow analysis.

Cohort Design This study was an investigator-initiated, prospective, observational cohort study of SAH patients admitted to the Neuro-ICU at BIDMC in Boston, Massachusetts. In patients from whom CSF was collected, SAH occurred between 2015 and 2016. With informed consent obtained from patients, or their legally authorized representative (LAR), cerebrospinal fluid (CSF) was collected from patients who had EVDs placed for clinical reasons within $48 \mathrm{~h}$ of ictus. The diagnosis of SAH was established by computed tomography (CT) or xanthochromia of the cerebrospinal fluid if the CT was negative. Patients were not enrolled if (1) aged less than 18 years old, (2) pregnant, (3) greater than $48 \mathrm{~h}$ since ictus, or (4) patients, families, or LARs did not want to participate in the study. All patients, or their LARs, that consented to this study were included. No informed consent was obtained for the 2010-2018 SAH cohort, where CSF was not collected, as all patient information was de-identified. All patient eligibility requirements, consent methodology, and enrollment protocols, as well as sample collection, processing, and storage procedures, were approved by the Institutional Review Board (IRB) of BIDMC.

General Management The care of SAH patients conformed to guidelines established by the American Heart Association [17].

Clinical and Radiographic Data Collection Clinical variables such as Hunt-Hess Grade [18], general demographics, patient history, laboratory values, culture data, and medications were recorded for the first week after admission to the BIDMC Neuro-ICU. Chest radiographs and venous ultrasounds were performed, per protocol if the patient was febrile, and read by radiologists blinded to the study. The dates of placement and discontinuation of central venous lines, Foley catheters, external ventriculostomy drains, and endotracheal tubes were all recorded. Admission CT scans were independently evaluated by a study neurointensivist for the presence and quantity of blood in the subarachnoid space and the intraventricular space to determine the modified Fisher $(\mathrm{mF})$ score $[19,20]$.

Determination of Subarachnoid Hemorrhage-Induced Pyrexia For a patient to have a fever defined as SAHiP, they had to fulfill 3 criteria. First, the patient had to have a temperature $>38.5^{\circ} \mathrm{C}$ during the first week of Neuro-ICU admission. Temperature is measured every $6 \mathrm{~h}$ on every patient in the Neuro-ICU. Second, the patient had to have negative cultures of the cerebrospinal fluid, blood, broncho-alveolar lavage, and urine over the first week of admission. Third, all chest radiographs and venous ultrasounds performed during the first week could not indicate an alternate source of fever.

\section{Protocol for Administration of Acetaminophen in Febrile SAH} Patients Patients with temperatures greater than $38.5^{\circ} \mathrm{C}$ during the first week of Neuro-ICU admission received $1 \mathrm{~g}$ of intravenous acetaminophen for a maximum of $4 \mathrm{~g}$ per day. If the fever decreased by at least $0.5^{\circ} \mathrm{C}$ at the next temperature check, acetaminophen administration was continued, if not it was halted. Opiates are not given for fever in our Neuro-ICU.

Determination of Delayed Cerebral Ischemia Delayed cerebral ischemia (DCI) was defined by two criteria. First, the cerebral infarction must not be evident on the admission CT scan. Second, it must not be a result of a surgical complication. CT scans at 7 days were evaluated for any evidence of DCI, by neuroradiologists blinded to the study. Ultimately, DCI was treated as a categorical variable, so if there was any evidence of an ischemic stroke that fulfilled DCI criteria, the CT scan was interpreted as positive.

Statistical Analysis Continuous variables were assessed for normality with skewness and kurtosis. Data that was not normally distributed was reported as medians with inter-quartile ranges. Data that was normally distributed was reported with means and standard deviations. Categorical variables were reported as count and proportions in each group. Grouping was based on clinical significance or median versus mean, depending on normality. To study univariate associations between SAHiP and other variables, chi-square or $t$ test analysis, for categorical versus continuous variables, were performed, respectively. Associations at $p<0.2$ from the univariate analysis, as well as variables with biological plausibility, were entered into the regressions, in a forward, stepwise manner using SPSS 25 to determine independent associations with SAHIP (SPSS Inc., Chicago, IL, USA). 


\section{Results}

No difference was seen between three male and three female wild-type (WT) SAH mouse models in terms of fever, and so the two genders were grouped together; that is, an $n$ of 6 . The temperature of WT control mice and WT SAH mice is shown (Fig. 1A). The temperature of the WT SAH mice is significantly higher at every time point $(p<0.04$ by 2 -way ANOVA). A common way to study fever in the Neuro-ICU patient population is to calculate a fever burden, or area under of the curve of fever with respect to time, and therefore the fever burden of SAH and control mice is shown in Fig. 1B $[11-13,21]$. Also seen in the figure is a positive control for fever using the canonical toll-like receptor 4 (TLR4) agonist, lipopolysaccharide (LPS), injected into the ventricular space. No difference was seen between fever and fever burden when comparing SAHiP to the LPS-induced fever.

To determine whether TLR4 was necessary for SAHiP, we repeated the above experiment placing data loggers in global $\mathrm{TLR}^{-/-}$mice and induced SAH after 1 week. The results demonstrate that global TLR4 ${ }^{-/-}$mice with SAH were profoundly hypothermic compared to SAH in wild-type mice
(Fig. 2A). We next wanted to investigate the role of the canonical fever pathway involving the neuronal prostaglandin EP3 receptor. Surprisingly, when SAH was induced in the Nestin ${ }^{\mathrm{Cre}}:$ ptger $3^{\mathrm{fl} / \mathrm{fl}}$ mice, their fever curve and fever burden was identical to that of WT SAH mice (Fig. 2B). Similarly when WT SAH mice were treated with meloxicam, a cyclooxygenase (COX) inhibitor, the fever curve and fever burden were unaffected (Fig. 2B).

To further elucidate the cell populations involved in SAHiP, we posited that microglial TLR4 or peripheral leukocyte TLR4 may be necessary. To test this hypothesis, we generated 2 novel, conditional knockout mice, Lyz ${ }^{\mathrm{Cre}}: \mathrm{TLR} 4^{\mathrm{fl} / \mathrm{fl}}$ and $\mathrm{CX} 3 \mathrm{Cr} 1^{\mathrm{CreER}}$ : $\mathrm{TLR} 4^{\mathrm{fl} / \mathrm{fl}}$ mice, where both Cre drivers have been previously described and validated [15, 22-24]. With respect to TLR4 expression, we validated that TLR4 was not expressed on microglia in the brain, or neutrophils in the peripheral blood in the Lyz ${ }^{\mathrm{Cre}}$ :TLR4 ${ }^{\mathrm{fl} / \mathrm{fl}}$ mice using flow cytometry (Fig. 3A). Similarly, we confirmed that TLR4 was not expressed on microglia in the brain, but was expressed on neutrophils in the peripheral blood of $\mathrm{CX} 3 \mathrm{Cr} 1^{\mathrm{CreER}}$ : TLR $4^{\mathrm{fl} / \mathrm{fl}}$ mice (Fig. 3B). Furthermore, we found no significant difference in fever burden between the TLR4 conditional knockout,
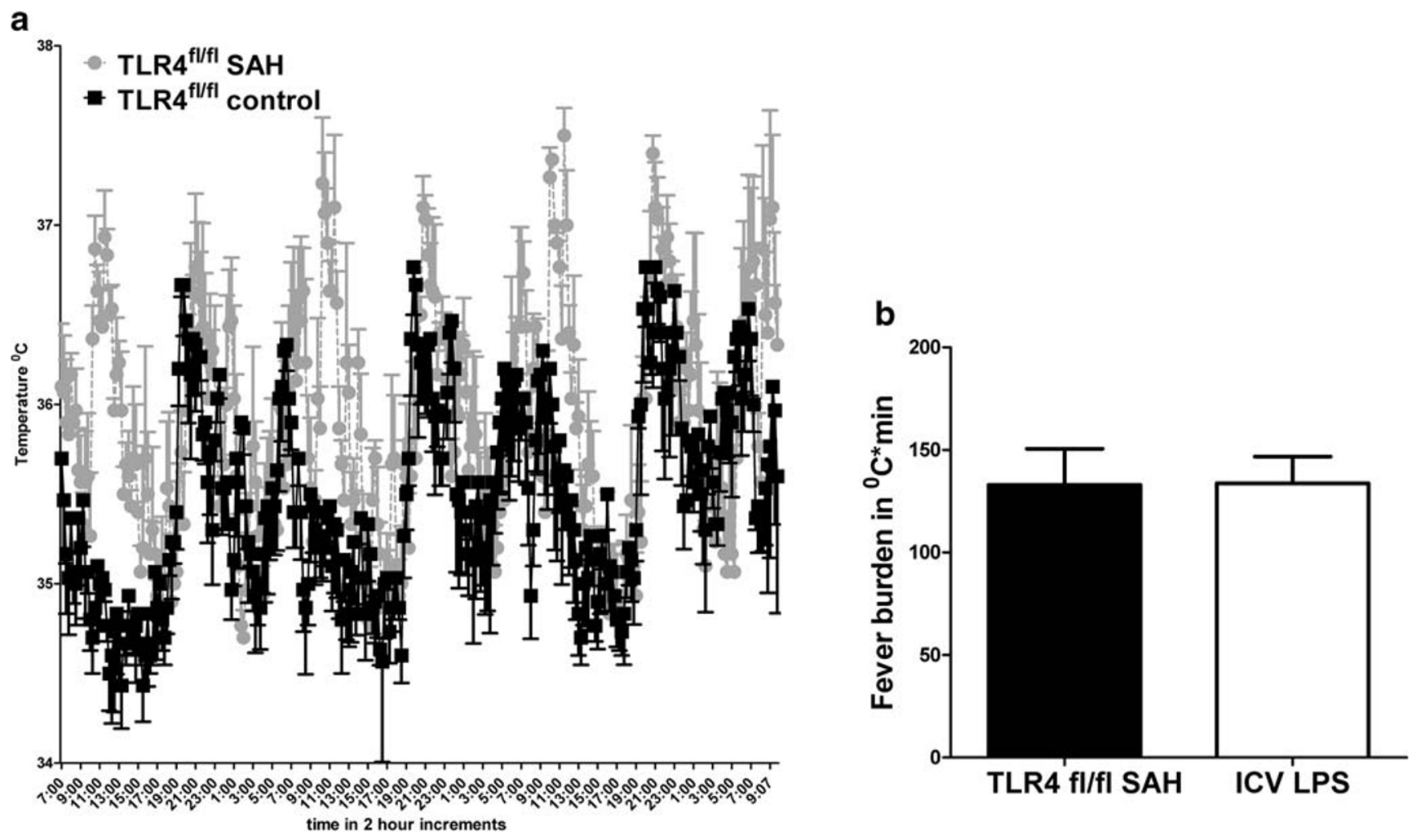

Fig. 1 (A) Intraperitoneal thermometers (dataloggers) were implanted in 8 male and 8 female TLR $4^{\mathrm{fl} / \mathrm{fl}}$ mice on C57BL/6 background. After 1 week, male $(n=4)$ and female $(n=4)$ mice were subjected to subarachnoid injection of $60 \mu \mathrm{l}$ of whole blood from CD45.1 $1^{+}$mice $(\mathrm{C} 57 \mathrm{BL} / 6$ background); the remaining 8 mice were subjected to subarachnoid injection of $60 \mu \mathrm{l}$ of normal saline. Post-SAH surgery ( $36 \mathrm{~h})$, temperatures were recorded from the two groups and are shown for every $8 \mathrm{~h}$, with mean and standard error of the mean. The mice subjected to SAH had significantly higher temperatures at all time points by two-way ANOVA ( $p<0.03 ; n=16$ ). (B) Fever burden was calculated by subtracting $36.5^{\circ} \mathrm{C}$ from each time point, $36 \mathrm{~h}$ after SAH surgery, and summing the values over 3.125 days, or 300 measurements at 15 -min intervals. Intracerebroventricular injection of $5 \mu \mathrm{g}$ of lipopolysaccharide was performed as a control for TLR4-dependent fever in male $(n=3)$ and female $(n=3) \mathrm{TLR}^{\mathrm{fl} / \mathrm{fl}}$ mice. Statistical significance was determined by Student's $t$ test and showed no difference 


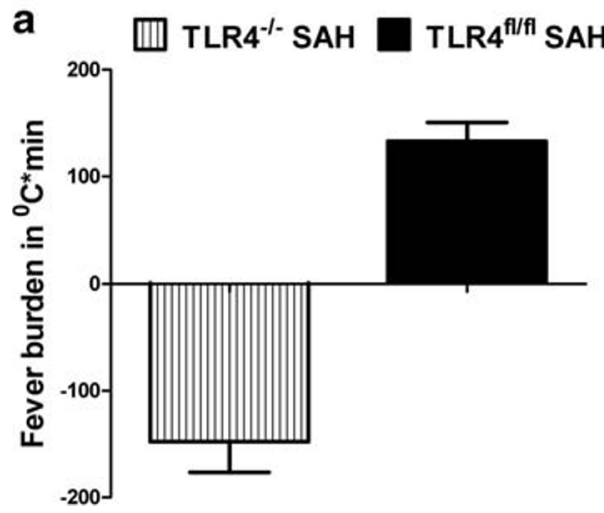

Fig. 2 (A) Fever burden in TLR $4^{\mathrm{fl} / \mathrm{fl}} \mathrm{SAH}$ mice was compared with the fever burden of global TLR $4^{-/-}$SAH mice. The lack of TLR 4 completely inhibited the SAHiP response. Statistical significance was determined by student's t test ( $p<0.0001 ; n=3 \mathrm{~F}, n=3 \mathrm{M})$. (B) Prostaglandin pathway involvement in SAHiP was determined by performing SAH in Nestin ${ }^{\text {Cre }}$ : ptger $3^{\mathrm{fl} / \mathrm{fl}}$ mice, which yielded the same fever burden as observed for

the global TLR4 knockout, and wild-type mice exposed to control normal saline injections (Fig. 3C).

We next repeated the SAHiP experiment in the novel conditional knockouts of TLR4 and found no significant difference between the hypothermia exhibited by the global TLR $4^{-1}$ SAH mice and $\mathrm{Lyz}^{\mathrm{Cre}}: \mathrm{TLR} 4^{\mathrm{fl} / \mathrm{fl}}$ or $\mathrm{CX} 3 \mathrm{Cr} 1^{\mathrm{CreER}}: \mathrm{TLR} 4^{\mathrm{fl} / \mathrm{fl}}$ SAH mice (Fig. 4).

We next wanted to examine whether cerebral macrophage TLR4 was involved in SAHiP seen in patients. We took cerebrospinal fluid (CSF) from 21 consecutive SAH patients. We then performed flow cytometry on the CSF leukocytes and identified the percentage of $\mathrm{CD} 14^{+} \mathrm{CD} 15^{-}$macrophages, where CD15 is a marker of neutrophils. An attempt was made to gate this population for TLR4, but there was no expression of this receptor or MD-2, the co-receptor of TLR4; however, CD14 is the other co-receptor of TLR4 required for downstream activation $[25,26]$. Because of the lack availability of any human antibodies that recognized TLR4 or MD-2, we used CD14 as a surrogate for TLR4 expression. We found a significant increase in $\mathrm{CD} 14^{+} \mathrm{CD} 15^{-}$macrophages in $\mathrm{SAH}$ patients with SAHiP versus those without SAHiP (Fig. 5).

We next wanted to validate independent associations with SAHiP in a large SAH patient population. Over the last 9 years, we enrolled 458 consecutive, de-identified, SAH patients in our database and using our electronic medical record system in the Neuro-ICU, kept track of demographics, radiography, chemistry, and infectious workup results. The general characteristics of the patient population can be seen in Table 1. Of note, we found that $28 \%$ of our population was febrile in the first week, although we defined a febrile threshold at $38.5{ }^{\circ} \mathrm{C}$ which could explain the lower incidence of fever in our Neuro-ICU. Furthermore, of SAH patients that were febrile, the majority of them had SAHiP.

To determine independent associations with SAHiP, a binary logistic regression was performed with SAHiP versus

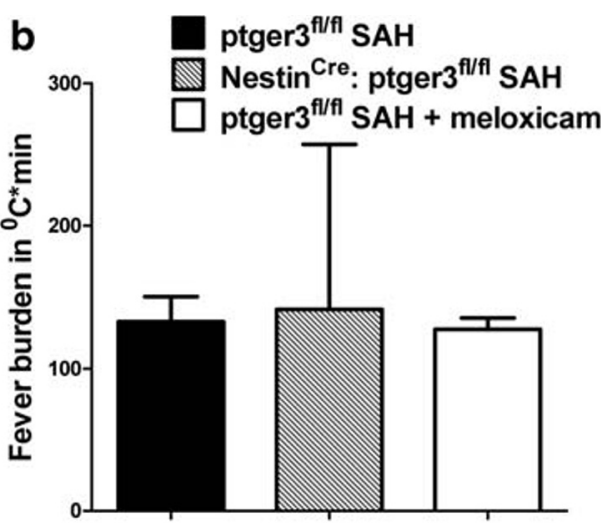

$\mathrm{TLR} 4{ }^{\mathrm{fl} / \mathrm{fl} 1} \mathrm{SAH}$ and ptger $3^{\mathrm{fl} / \mathrm{fl}}+100 \mu \mathrm{g}$ meloxicam SAH mice. The SAHiP response was unaffected by the total lack of prostaglandin EP3 receptors in neurons, and the inhibition of the cyclo-oxygenase enzyme by meloxicam. Statistical significance was determined by two-way ANOVA and showed no differences $(n=6$ per group, equally divided between the genders)

non-SAHiP fever as the dependent, categorical variable. Three variables were independently, and negatively, associated with SAHiP: acetaminophen administration, duration of central line placement in hours, and age in years. That is, SAH patients that do not receive acetaminophen, those with central lines in place for shorter times, and those that are younger are more likely to have SAHiP (Table 2).

\section{Discussion}

Fever in patients with SAH likely has a very different mechanism by which it occurs. From mouse models, it has long been established that infectious fever, using peripheral lipopolysaccharide (LPS) injection, occurs via a cyclo-oxygenase (COX)-dependent mechanism which relies on the production of prostaglandins [7-10, 27-29]. The peripheral LPS injection activates TLR4 on leukocytes and endothelial cells, resulting in the production of PGE2, which then diffuses into the brain. The PGE2 then binds to and activates the prostaglandin EP3 receptor in the median preoptic nucleus of the hypothalamus, which then results in a fever by canonical mechanisms. This also explains why pharmaceuticals like acetaminophen, meloxicam, or other COX inhibitors are effective in suppressing these canonical fevers, which we will term classical fever. As such, terms such as neurogenic or central fever are redundant as all fever requires a neurological relay, whether it is classical fever or SAHiP.

In SAHiP, whether it is in an SAH mouse model or SAH patients, there is no source of fever at all. While heme and other hemoglobin byproducts can be TLR4 ligands $[14,30$, 31], similar to LPS, the location of TLR4 stimulation, and the downstream signal transduction, is very different between classical fever and SAHiP. In classical fever, TLR4 stimulation first occurs peripherally with circulating 


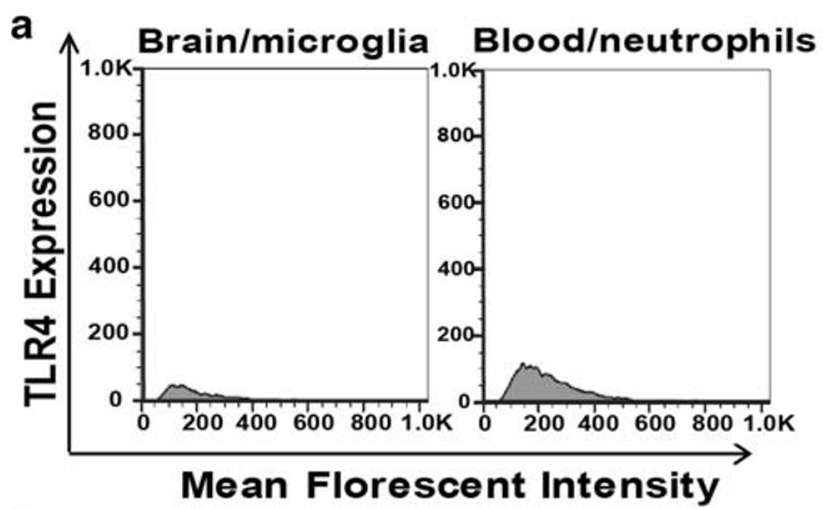

b

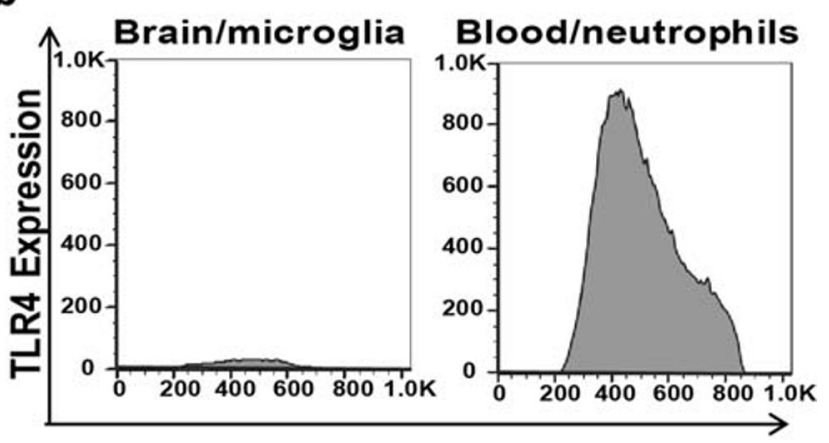

Mean Florescent Intensity

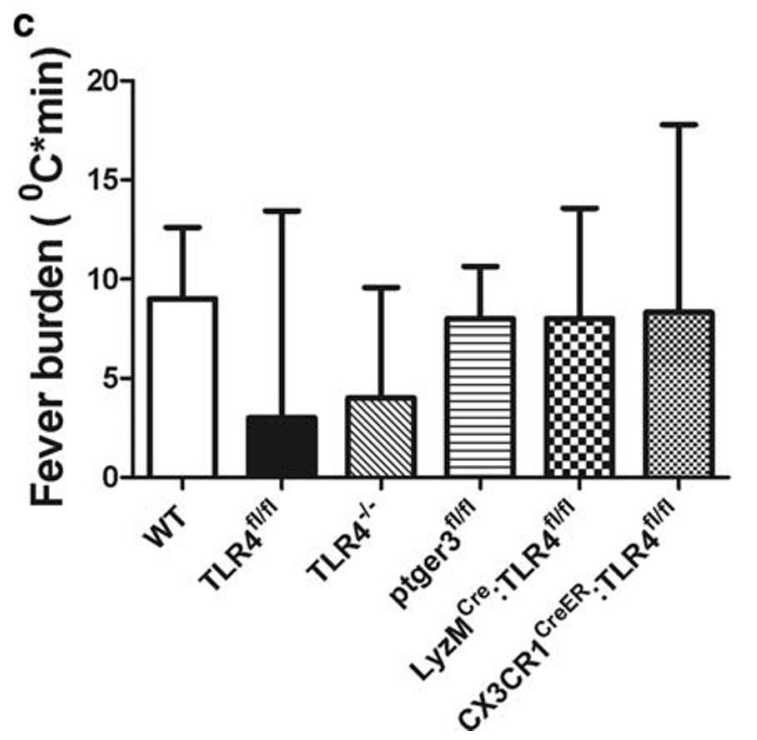

macrophages and neutrophils, as well as peripheral endothelial cells, which result in production of PGE2 [28]. Conversely, in SAHiP, based on our SAH mouse model, stimulation of microglial TLR4 is necessary for SAHiP, and no peripheral stimulation of TLR4 on circulating macrophages and neutrophils is required (Fig. 4). Furthermore, our lab, as well as others, has shown that the TLR4 activation by heme versus LPS results in very different downstream signal transduction $[14,32]$. There is evidence for this in the SAH mouse model, as well as clinically in SAH patients. First, the classical fever pathway requires the
Fig. 3 (A) Flow cytometry of brain homogenates and peripheral blood obtained from LyzM ${ }^{\mathrm{Cre}}:$ TLR $4^{\mathrm{fl} / \mathrm{fl}} \mathrm{SAH}$ mice was performed. Results are illustrated by the representative histogram. Blood injected into the brain was excluded from analysis by $\mathrm{CD} 45.1^{+}$selection, leaving only endogenous CD45.2 $2^{+}$cells. Microglia were sequentially gated: CD $45.2^{+} \mathrm{CD} 11 \mathrm{~b}{ }^{\text {hi }} \mathrm{CX} 3 \mathrm{CR} 11^{\text {hi }} \mathrm{CD} 68^{\text {int }} \mathrm{Tmem} 119^{\text {hi }}$. This population was interrogated for TLR4 expression, which is represented by the brain/ microglia histogram. Circulating monocytes and neutrophils were gated by size and granularity (forward and side scatter), and by CD $11 b^{\text {hi }} \mathrm{CD} 68^{\text {hi }}$ and $\mathrm{Gr}-1^{\text {hi }}$. As for the peripheral blood, TLR4 expression was subsequently interrogated and is represented by the blood/neutrophils histogram. Data demonstrated that TLR4 was not expressed in either microglia, or neutrophils $(n=3)$. (B) Similar flow and gating procedures were performed on the $\mathrm{CX}_{3} \mathrm{Cr} 1^{\text {CreER }}$ :TLR $4^{\mathrm{fl} / \mathrm{fl}} \mathrm{SAH}$ mice. A representative histogram is shown. Data illustrated a lack of TLR4 expression in microglia, but normal expression of TLR4 in circulating neutrophils $(n=3$ mice). (C) The fever burdens for wild-type C57BL/6 (WT), TLR $4^{\mathrm{fl} / \mathrm{fl}}$, $\mathrm{TLR}^{-1-}$, ptger $^{\mathrm{fl} / \mathrm{fl}}, \mathrm{LyzM}^{\mathrm{Cre}}$ :TLR4 $4^{\mathrm{fl} / \mathrm{fl}}$, and $\mathrm{CX}_{3} \mathrm{Cr}^{\mathrm{CreER}}:$ TLR4 ${ }^{\mathrm{fl} / \mathrm{fl}}$ control mice injected with $60 \mu \mathrm{l}$ of normal saline are shown with mean and standard error of the mean. All mice were injected with tamoxifen 1 week prior to the recording. Statistical significance was determined by one-way ANOVA and showed no differences between fever burdens of control NS injections between mice of different genotypes ( $n=3$ per group)

prostaglandin EP3 receptor in the median preoptic nucleus, which is deleted in the Nestin ${ }^{\mathrm{Cre}}:$ ptger $3^{\mathrm{fl} / \mathrm{fl}}$ mice [9]. Mice, exposed to $\mathrm{SAH}$ with this prostaglandin receptor deletion still had SAHiP that was unchanged from wild-type mice (Fig. 2). Further, SAHiP in WT SAH mice was unaffected by meloxicam treatment (Fig. 2). Therefore, even if heme or its byproducts activate TLR4, then COX, and result in subsequent PGE2 production, PGE2 is not required for SAHiP. In addition to our mouse model of SAHiP, there is indirect evidence for this in SAH patients, as well.

In 21 febrile SAH patients where cerebrospinal fluid (CSF) was collected and subjected to flow cytometry, patients with more $\mathrm{CD} 14^{+} \mathrm{CD} 15^{-}$macrophages in their CSF were more likely to have SAHiP. Although the number of patients is very small, this again suggests a different immunological mechanism for SAHiP versus classical fever in SAH patients. While we could not show that TLR4 expression on CSF CD14 $4^{+} \mathrm{CD} 15^{-}$macrophages was critical to SAHiP, due to lack of selective human anti-TLR4 flow cytometry antibodies; CD14 is in fact a necessary component to TLR4 signaling $[25,26,33]$. CD15 is a specific marker for neutrophils in humans, so the $\mathrm{CD} 14^{+} \mathrm{CD} 15^{-}$subset of macrophages likely all express TLR4 and support our conclusion that cerebral macrophage TLR4 is necessary for SAHiP compared to classical fever.

Finally, our logistic regression of $129 \mathrm{SAH}$ patients with fever revealed an inverse relationship between acetaminophen administration and propensity for SAHiP. This could indicate that intensivists that administer acetaminophen do not see an effect on SAHiP, whereas they do see an effect with classical fever and so continue to administer acetaminophen to these patients.

An interesting corollary of our findings might address the apparent effectiveness of bromocriptine in the treatment of SAHiP [34]. One in vitro study suggested that microglial 
Fig. 4 Determination of whether microglial TLR4, neutrophil

TLR4, or both were necessary for SAHiP. SAH was induced in global TLR4 ${ }^{-/}$, microglial $\mathrm{TLR}^{-/-}\left(\mathrm{CX}_{3} \mathrm{Cr}^{\mathrm{CreER}}: \mathrm{TLR}^{\mathrm{fl} / \mathrm{fl}}\right.$ mice), and mice that lacked TLR4 on microglia and all peripheral myeloid cells $\left(\right.$ LyzM $^{\mathrm{Cre}}:$ TLR $4^{\mathrm{fl} / \mathrm{fl}}$ mice). The fever burden in all murine subsets were determined to be the same and indicated that only microglial TLR4 was necessary to mediate SAHiP. Statistical significance was determined by ANOVA and showed no differences $(n=6$ per group, equally divided between the genders)

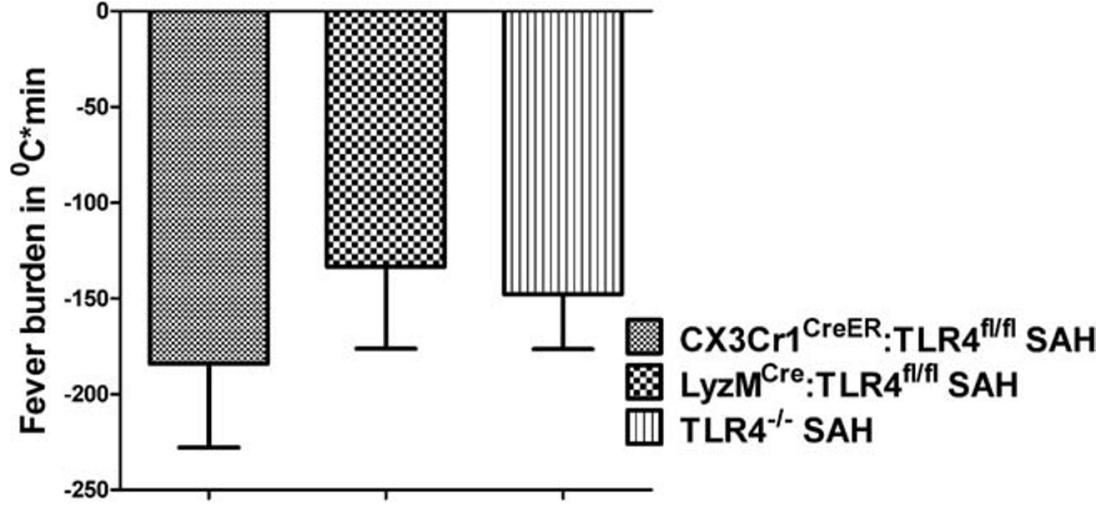

cultures exposed to $\alpha$-synuclein, a protein aggregate found in Parkinson's disease, release glutamate via TLR2 and TLR4 activation [35]. The authors also found that glutamate release can be prevented by dopamine. Interestingly, the effector pathway of both classical fever and likely SAHiP requires glutamate neurotransmission from the rostral medullary raphe to induce fever [7, 28]. Perhaps bromocriptine, via dopamine agonism, mediates its effects in SAHiP by inhibiting microglial glutamate release induced by heme activation of TLR4. In the future, this could be investigated by cerebral

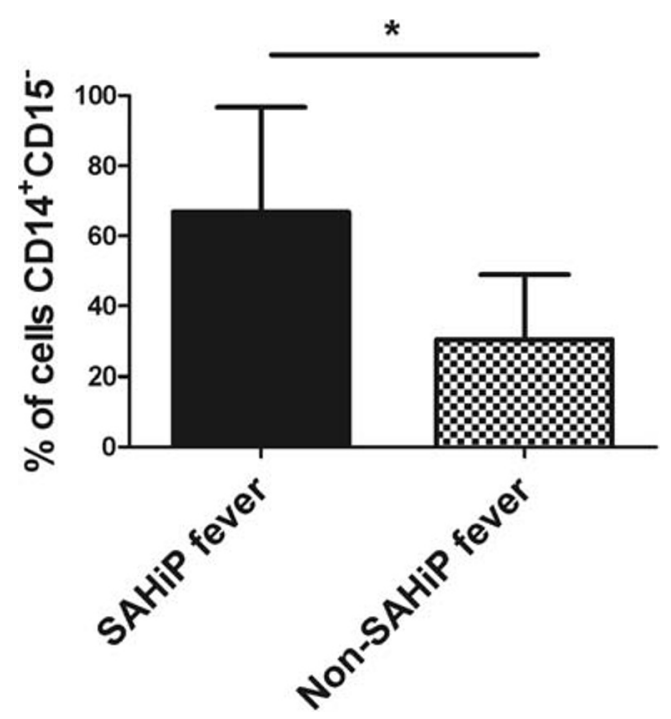

Fig. 5 CSF obtained from SAH patients was subjected to flow cytometry and interrogated for $\mathrm{CD} 14^{+} \mathrm{CD} 15^{-}$macrophages. Patients were divided into those with SAHiP and non-SAHiP fever, and the percent population of macrophages is shown. Statistical significance was determined by Student's $t$ test $p<0.03$, and showed that patients with higher percentage of CSF macrophages had more SAHiP ( $n=21 \mathrm{SAH}$ patients) microdialysis of both SAH patients and rodent models that exhibit SAHiP after administration of bromocriptine.

One other study has examined SAHiP-like fevers, which were termed neurogenic fevers by the authors [6]. There were several important differences between this previous study and our current study. The authors did include venous thromboembolism as one cause of non-infectious fevers, but not atelectasis, like our study did. Furthermore, our study was restricted to $\mathrm{SAH}$, whereas this previous study included all Neuro-ICU patients. Because we defined SAHiP based on the lack of growth from any bodily fluid culture, no infiltrate or atelectasis on chest radiograph, and no venous thrombosis, we did not use these as independent variables. We did control for blood transfusions, but perhaps due to differences in practice and different patient populations, only 8 patients or $6 \%$ of our febrile SAH patients received any form of blood transfusion; no association was found on univariate or multivariate analysis between SAHiP and blood transfusion. We also looked at the onset of SAHiP versus non-SAHiP fever and found that both occurred with approximately $72 \mathrm{~h}$ of Neuro-ICU admission. We also examined the association of intraventricular hemorrhage (IVH) and SAHiP, since the modified Fisher score for SAH accounts for IVH and we found no association between IVH in SAH and SAHiP. The differences in between our studies can be explained by a host of reasons. In addition to the mixed ICU population and the differing definition of fever, the administration of acetaminophen was protocolized in this previous study, and not controlled for as an independent variable. The inverse independent association with acetaminophen we found was likely due to the fact that our data demonstrate that SAHiP is mediated via non-COX mechanisms. 
Table 1 Patient Characteristics

\begin{tabular}{|c|c|c|}
\hline & Total SAH patient population & Subset of febrile SAH patients \\
\hline Number & 458 & 129 \\
\hline \multicolumn{3}{|l|}{ Age (years) } \\
\hline Male & $55(42-68)$ & $55(42-68)$ \\
\hline Female & $60(45-75)$ & $62(50-74)$ \\
\hline Male sex $(\%)$ & $185(38 \%)$ & $56(43 \%)$ \\
\hline Modified Fisher grade & $3(2-4)$ & $3(2-4)$ \\
\hline Hunt and Hess grade & $3(2-5)$ & $3(2-5)$ \\
\hline Aneurysm identified & $294(64 \%)$ & $98(76 \%)$ \\
\hline Delayed cerebral ischemia & $37(19 \%)$ & $24(19 \%)$ \\
\hline Hypertension & $248(54 \%)$ & $65(50 \%)$ \\
\hline Migraine & $69(15 \%)$ & $9(7 \%)$ \\
\hline Alcohol & $358(78 \%)$ & $91(70 \%)$ \\
\hline Smoking & $321(70 \%)$ & $41(32 \%)$ \\
\hline Coiled & $271(59 \%)$ & $84(65 \%)$ \\
\hline Red blood cell transfusion & $45(10 \%)$ & $8(6 \%)$ \\
\hline Duration of endotracheal tube (days) & $3(1-14)$ & $5(3-18)$ \\
\hline Duration of Foley catheter (days) & $6(3-14)$ & $8(7-13)$ \\
\hline Duration of external ventricular drain (days) & $7(6-14)$ & $7(6-14)$ \\
\hline Duration of central venous access (days) & $7(3-10)$ & $9(7-11)$ \\
\hline Acetaminophen administered & $99(22 \%)$ & $48(37 \%)$ \\
\hline Acetaminophen administered (grams) & $1(0-2)$ & SAHiP 5 (2-8) non-SAHiP 14 (6-22) \\
\hline SAHiP patients & $66(14 \%)$ & $66(52 \%)$ \\
\hline ICU length of stay (days) & $9(5-14)$ & $12(7-15)$ \\
\hline Modified Rankin at last follow-up & $2(1-6)$ & $2(2-6)$ \\
\hline
\end{tabular}

In summary, our findings indicate a novel mechanism for SAHiP that is independent of COX and dependent on microglial TLR4 in a mouse model of SAH. Support for the dependence on macrophage TLR4 is seen in the flow
Table 2 Univariate and multivariate analysis for independent association with SAHiP

\begin{tabular}{|c|c|c|c|}
\hline & \multirow{2}{*}{$\begin{array}{l}\text { Univariate }(n=129) \\
p \text { value }\end{array}$} & \multicolumn{2}{|c|}{ Multivariate logistic $(n=129)$} \\
\hline & & OR $(95 \% \mathrm{CI})$ & $p$ value \\
\hline Age & 0.18 & $0.95(0.96-0.98)$ & $<0.04$ \\
\hline Gender & 0.38 & Included & 0.73 \\
\hline Hunt and Hess grade & 0.34 & Included & 0.38 \\
\hline Modified Fisher score & 0.78 & Included & 0.6 \\
\hline Delayed cerebral ischemia & 0.29 & & \\
\hline Smoking & 0.3 & & \\
\hline Hypertension & 0.3 & & \\
\hline Acetaminophen & 0.2 & $0.21(0.12-0.31)$ & $<0.001$ \\
\hline Blood transfusion & 0.6 & & \\
\hline Foley catheter duration (hours) & 0.11 & Included & 0.62 \\
\hline Endotracheal tube duration (hours) & 0.55 & & \\
\hline External ventriculostomy duration (hours) & 0.4 & & \\
\hline Central venous line duration (hours) & 0.035 & $0.98(0.98-0.99)$ & $<0.05$ \\
\hline Surgical management & 0.4 & & \\
\hline
\end{tabular}

The italics indicate that the variable was included in the multivariate logistic regression 
cytometry results of SAH patients with SAHiP, and further corroborated by the inverse, independent association with acetaminophen in a large cohort of febrile SAH patients.

Acknowledgements Dr. Hanafy receives support from the National Institute of Neurological Disorders and Stroke (R21NS099606 and R01NS109174) and the American Heart Association Grant in Aid (17GRNT33670058). I would like to thank Ariel Mueller for her help in data acquisition.

Author Contributions AJT, LAC, SG, MS, and GM were involved in acquisition and analysis of data. KAH was involved in conception and design of the study, analysis of data, and drafting a significant portion of the manuscript and figures. All authors consent to this publication.

\section{Compliance with Ethical Standards}

Conflict of Interest The authors declare that they have no conflict of interest.

\section{References}

1. Commichau C, Scarmeas N, Mayer SA. Risk factors for fever in the neurologic intensive care unit. Neurology 2003;60(5):837-841.

2. Diringer MN, Reaven NL, Funk SE, Uman GC. Elevated body temperature independently contributes to increased length of stay in neurologic intensive care unit patients. Crit Care Med 2004;32(7):1489-1495.

3. Hanafy KA, Morgan Stuart R, Fernandez L, et al. Cerebral inflammatory response and predictors of admission clinical grade after aneurysmal subarachnoid hemorrhage. J Clin Neurosci 2010;17(1):22-25.

4. Kilpatrick MM, Lowry DW, Firlik AD, et al. Hyperthermia in the neurosurgical intensive care unit. Neurosurgery 2000;47(4):850 855; discussion 855-856.

5. Stocchetti N, Rossi S, Zanier ER, et al. Pyrexia in head-injured patients admitted to intensive care. Intensive Care Med 2002;28(11):1555-1562.

6. Hocker SE, Tian L, Li G, et al. Indicators of central fever in the neurologic intensive care unit. JAMA Neurol 2013;70(12):1499_ 1504.

7. Machado NLS, Abbott SBG, Resch JM, et al. A Glutamatergic Hypothalamomedullary Circuit Mediates Thermogenesis, but Not Heat Conservation, during Stress-Induced Hyperthermia. Curr Biol 2018;28(14):2291-2301.e5.

8. Yoshida K, Li X, Cano G, et al. Parallel preoptic pathways for thermoregulation. J Neurosci 2009;29(38):11954-11964.

9. Lazarus M, Yoshida K, Coppari R, et al. EP3 prostaglandin receptors in the median preoptic nucleus are critical for fever responses. Nat Neurosci 2007;10(9):1131-1133.

10. Lu J, Zhang YH, Chou TC, et al. Contrasting effects of ibotenate lesions of the paraventricular nucleus and subparaventricular zone on sleep-wake cycle and temperature regulation. J Neurosci 2001;21(13):4864-4874.

11. Broessner G, Beer R, Lackner P, et al. Prophylactic, endovascularly based, long-term normothermia in ICU patients with severe cerebrovascular disease: bicenter prospective, randomized trial. Stroke 2009;40(12):e657-665.

12. Diringer MN, Neurocritical Care Fever Reduction Trial Group. Treatment of fever in the neurologic intensive care unit with a catheter-based heat exchange system. Crit Care Med 2004;32(2): $559-564$.
13. Mayer SA, Kowalski RG, Presciutti M, et al. Clinical trial of a novel surface cooling system for fever control in neurocritical care patients. Crit Care Med 2004;32(12):2508-2515.

14. Hanafy KA. The role of microglia and the TLR4 pathway in neuronal apoptosis and vasospasm after subarachnoid hemorrhage. J Neuroinflammation 2013;10:83.

15. LeBlanc RH, Chen R, Selim MH, Hanafy KA. Heme oxygenase-1mediated neuroprotection in subarachnoid hemorrhage via intracerebroventricular deferoxamine. J Neuroinflammation 2016;13(1):244

16. Bennett ML, Bennett FC, Liddelow SA, et al. New tools for studying microglia in the mouse and human CNS. Proc Natl Acad Sci U S A 2016;113(12):E1738-1746.

17. Connolly ES, Rabinstein AA, Carhuapoma JR, et al. Guidelines for the management of aneurysmal subarachnoid hemorrhage: a guideline for healthcare professionals from the American Heart Association/american Stroke Association. Stroke 2012;43(6): 1711-1737.

18. Hunt WE, Hess RM. Surgical risk as related to time of intervention in the repair of intracranial aneurysms. J Neurosurg 1968;28(1):14 20.

19. Claassen J, Bernardini GL, Kreiter K, et al. Effect of cisternal and ventricular blood on risk of delayed cerebral ischemia after subarachnoid hemorrhage: the Fisher scale revisited. Stroke 2001;32(9):2012-2020.

20. Kramer AH, Hehir M, Nathan B, et al. A comparison of 3 radiographic scales for the prediction of delayed ischemia and prognosis following subarachnoid hemorrhage. J Neurosurg 2008;109(2): 199-207.

21. Naidech AM, Bendok BR, Bernstein RA, et al. Fever burden and functional recovery after subarachnoid hemorrhage. Neurosurgery 2008;63(2):212-217; discussion 217-218.

22. Schallner N, Pandit R, LeBlanc R, et al. Microglia regulate blood clearance in subarachnoid hemorrhage by heme oxygenase-1. J Clin Invest 2015;125(7):2609-2625.

23. Parkhurst CN, Yang G, Ninan I, et al. Microglia promote learningdependent synapse formation through brain-derived neurotrophic factor. Cell 2013;155(7):1596-1609.

24. Goldmann T, Wieghofer P, Müller PF, et al. A new type of microglia gene targeting shows TAK1 to be pivotal in CNS autoimmune inflammation. Nat Neurosci 2013;16(11):1618-1626.

25. Zanoni I, Ostuni R, Marek LR, et al. CD14 controls the LPSinduced endocytosis of Toll-like receptor 4. Cell 2011;147(4): 868-880.

26. Tan Y, Zanoni I, Cullen TW, et al. Mechanisms of Toll-like Receptor 4 Endocytosis Reveal a Common Immune-Evasion Strategy Used by Pathogenic and Commensal Bacteria. Immunity 2015;43(5):909-922.

27. Oka T, Oka K, Kobayashi T, et al. Characteristics of thermoregulatory and febrile responses in mice deficient in prostaglandin EP1 and EP3 receptors. J Physiol 2003;551(Pt 3):945-954.

28. Saper CB, Romanovsky AA, Scammell TE. Neural Circuitry Engaged by Prostaglandins during the Sickness Syndrome. Nat Neurosci 2012;15(8):1088-1095.

29. Steiner AA, Chakravarty S, Rudaya AY, et al. Bacterial lipopolysaccharide fever is initiated via Toll-like receptor 4 on hematopoietic cells. Blood 2006;107(10):4000-4002.

30. Figueiredo RT, Fernandez PL, Mourao-Sa DS, et al. Characterization of heme as activator of Toll-like receptor 4. J Biol Chem 2007;282(28):20221-20229.

31. Kwon MS, Woo SK, Kurland DB, et al. Methemoglobin is an endogenous toll-like receptor 4 ligand-relevance to subarachnoid hemorrhage. Int J Mol Sci 2015;16(3):5028-5046.

32. Sansing LH, Harris TH, Welsh FA, et al. Toll-like receptor 4 contributes to poor outcome after intracerebral hemorrhage. Ann Neurol 2011;70(4):646-656. 
33. Antal-Szalmas P, Strijp JA, Weersink AJ, et al. Quantitation of surface CD14 on human monocytes and neutrophils. J Leukoc Biol 1997;61(6):721-728.

34. Natteru P, George P, Bell R, et al. Central Hyperthermia Treated with Bromocriptine. Case Rep Neurol Med 2017;2017:1712083.
35. Dos-Santos-Pereira M, Acuña L, Hamadat S, et al. Microglial glutamate release evoked by $\alpha$-synuclein aggregates is prevented by dopamine. Glia 2018;66(11):2353-2365.

Publisher's Note Springer Nature remains neutral with regard to jurisdictional claims in published maps and institutional affiliations. 